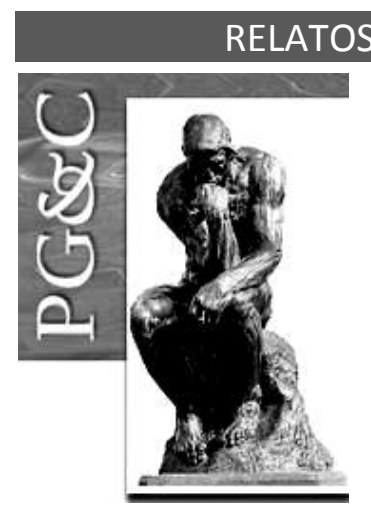

\title{
AUTOGERENCIAMENTO E ESTABELECIMENTO DE METAS NO TRABALHO: UM PANORAMA DA PRODUÇÃO CIENTÍFICA BRASILEIRA
}

\author{
Luiz Carlos Victorino \\ Doutorando em Psicologia pela Universidade de Brasília, Brasil. \\ Professor do Centro Universitário de Brasília, Brasil. \\ E-mail: luiz.victorino1@gmail.com \\ Rodrigo Rodrigues de Souza \\ Doutorando em Psicologia pela Universidade de Brasília, Brasil. \\ Professor do Centro Universitário de Brasília, Brasil. \\ E-mail: rodrigo rodrigues96@yahoo.com.br \\ Lígia Abreu Cruz \\ Doutoranda em Psicologia pela Universidade de Brasília, Brasil. \\ Professora do Centro Universitário de Brasília, Brasil. \\ E-mail: ligiaabreugc@gmail.com
}

\begin{abstract}
Resumo
O autogerenciamento e os objetivos no trabalho são temas relativamente comuns nas discussões em administração e psicologia organizacional. Contudo, a produção científica brasileira parece não demonstrar o mesmo interesse pelo tema, com poucos artigos publicados no campo do comportamento organizacional, que tenham como objetivo a investigação desses construtos. O objetivo do presente artigo foi realizar uma análise da produção científica brasileira em autogerenciamento e objetivos no trabalho nos últimos 21 anos. Este estudo revisou 16 relatos de pesquisas empíricas que abordam os temas supracitados, publicados em periódicos nacionais. Os resultados demonstraram uma grande divisão entre artigos que investigam o tema sob uma perspectiva mais macroscópica, com foco no autogerenciamento da carreira, e aqueles que, em uma perspectiva mais microscópica, investigam os comportamentos cotidianos dos trabalhadores. A maior parte dos estudos é exploratória, com surveys conduzidas por psicólogos doutores no setor terciário da economia e com dados primários. Esses resultados nos permitem caracterizar a produção científica defasada e ainda sem muito foco no Brasil.
\end{abstract}

Palavras-chave: Autogerenciamento. Objetivos no trabalho. Produção científica.

\section{SELF-MANAGEMENT AND GOAL-SETTING: AN OVERVIEW OF BRAZILIAN SCIENTIFIC PRODUCTION}

\begin{abstract}
Self-management and objectives at work are relatively common themes in discussions on administration and organizational psychology. However, the Brazilian scientific production does not seems to show the same interest in the subject, with few articles published in the field of organizational behavior regarding these constructs. The purpose of this article was to analyze the Brazilian scientific production in selfmanagement and objectives at work in the last 21 years. The present analysis reviewed 16 empirical research reports that address the above issues, published in national journals. The results showed a division between articles that investigate through a macroscopic perspective, focusing on career selfmanagement, and articles in a more microscopic perspective, that investigate the everyday behavior of workers. Most studies are exploratory, with surveys conducted by PhD psychologists in the tertiary sector of the economy, using primary data. These results allow us to characterize the Brazilian scientific production as lagged and still without focus.
\end{abstract}

Keywords: Self-management. Objectives at work. Scientific production.

Perspectivas em Gestão \& Conhecimento, João Pessoa, v. 8, n. 3, p. 243-254, set./dez. 2018. DOI: http://dx.doi.org/10.21714/2236-417X2018v8n3p243

http://periodicos.ufpb.br/ojs2/index.php/pgc. ISSN: 2236-417X. Publicação sob Licença (cc) EY-NC-ND 


\section{INTRODUÇÃO}

O comportamento organizacional (CO) é um dos campos de estudo centrais em psicologia organizacional, sendo caracterizado pela investigação dos comportamentos dos indivíduos e de grupos de indivíduos no âmbito das organizações (SOBRAL; MANSUR, 2013). Enquanto área multidisciplinar por definição, o estudo do comportamento organizacional atrai o interesse de muitos pesquisadores (BORGES-ANDRADE; PAGOTTO, 2010; HANNECK; SANTOS, 2018; DE CAMPOS; RUEDA, 2017; PODSAKOFF; MACKENZIE; PODSAKOFF, 2018; GELFAND, 2017) não apenas por perpassar uma infinidade de fenômenos nas organizações, mas também por sua interface com diferentes áreas do saber, como psicologia, administração e sociologia.

As principais características estruturantes da área, além de sua multi e interdisciplinaridade, são a tensão entre conhecimento, prática e os diferentes níveis de análise. Os problemas e questões que desafiam organizações, gestores, trabalhadores e formuladores de políticas públicas laborais exigem a compreensão de processos psicológicos no âmbito do trabalho. Ao mesmo tempo, as questões teórico-práticas podem ser divididas em múltiplos níveis (individual, grupal, social e governamental) (NEIVA; CORRADI, 2011). A diversidade e a ampliação da área decorrem da sua inserção como campo de aplicação e pela pressão contínua das demandas sociais e organizacionais, facilitando a incorporação de novos tópicos de pesquisa para dar subsídios à atuação profissional.

Uma grande contribuição para a compreensão deste panorama são as revisões de literatura conduzidas a partir do modelo adotado por Borges-Andrade e Pagotto (2010), que permitem uma visão mais ampla do campo e suas possibilidades de desenvolvimento. Os dados dos pesquisadores supracitados apresentam, por exemplo, algumas características como a predominância de pesquisas com delineamento, realizadas no setor público, investigando afetos no trabalho, aprendizagem e interações sociais como características básicas das publicações em $\mathrm{CO}$ no Brasil.

Apesar de historicamente a área de psicologia do trabalho e dar organizações ter se desenvolvido na América latina com forte influência europeia, com o desenvolvimento da área ao longo dos anos, as agendas de pesquisa foram se distanciando, não apenas como fruto dos fatores internos de cada país, mas também pelo próprio aumento da complexidade da área (BORGES-ANDRADE; PEREZ; TORO, 2018).

Deste modo, o objetivo da presente revisão sistemática de literatura é compreender o atual estado das pesquisas brasileiras em autogerenciamento e objetivos no trabalho, para traçar um panorama em comparação à produção nacional em $\mathrm{CO}$ e, a partir daí, apontar caminhos para o desenvolvimento de uma agenda de pesquisa na área.

\section{REFERENCIAL TEÓRICO}

A exigência de desempenho tem se tornado um elemento cada vez mais presente nas organizações modernas, com indivíduos sendo cada vez mais cobrados e com parâmetros de performance cada vez mais altos. Nesse sentido, apesar da busca por desenvolvimento ser uma característica fundamental da natureza humana, ainda perduram questões centrais no que se refere a como esses processos de estabelecimento e alcance de metas se desenrolam.

O interesse científico sobre os processos cognitivos no âmbito do trabalho é uma tendência relativamente estável na pesquisa internacional, com estudos sobre inteligência e motivação sendo publicados desde os anos 70, até os estudos mais recentes sobre aprendizagem, autorregulação e metas (BORGES-ANDRADE; PEREZ; TORO, 2018).

Além desse aspecto, mudanças em termos de métodos também ocorreram nos últimos anos, especialmente se considerarmos que, por exemplo, os estudos sobre motivação que eram em geral conduzidos a partir de uma perspectiva interpessoal, recentemente passaram a

Perspectivas em Gestão \& Conhecimento, João Pessoa, v. 8, n. 3, p. 243-254, set./dez. 2018. 
ser abordados sob um ponto de vista longitudinal e intrapessoal. As mudanças têm obrigado os pesquisadores a adotar novos métodos e técnicas de análise, além de incorporar conhecimentos de pesquisas básicas em cognição (LORD; DIEFENDORFF; SCHMIDT; HALL, 2010).

Em âmbito internacional, as pesquisas sobre autogerenciamento e objetivos no trabalho tem se diversificado e avançado bastante em termos de sofisticação metodológica e integração de conhecimentos de outras áreas. Os estudos abordam não apenas a performance em tarefas, como tradicionalmente ocorria, mas também investigam os efeitos destes construtos em áreas como a cidadania organizacional, a efetividade dos trabalhadores, as estratégias para lidar com mudanças organizacionais e até mesmo os efeitos dos grupos sociais e de características socioculturais (CAVALLO; HOLMES; FITZSIMONS; MURRAY; WOOD, 2012; JENSEN; RAVER, 2012; STURGES; CONWAY; LIEFOOGHE, 2010).

Uma meta-análise que investigou o atingimento de metas auto-reportadas e o desempenho nas áreas de trabalho, esportes e educação (VAN YPEREN, BLAGA \& POSTMES, 2014) apontou que boa parte da literatura nesta área tem apresentado uma tendência mais a fragmentação do que a integração, o que dificulta por exemplo que os achados em uma determinada área possam ser extrapolados para outras. Um dos principais resultados deste estudo apontou, por exemplo, que no âmbito do trabalho e da educação, a chance de sucesso no atingimento das metas era maior quando as metas estabelecidas eram no campo da prevenção, mais do que da promoção, a partir da perspectiva da teoria do foco regulatório (KRUGLANSKI et al., 2000), mas tais resultados não podiam ser extrapolados para o âmbito do esporte.

Os autores da meta análise sugerem exatamente que esforços sejam empreendidos no sentido de não apenas tentar integrar a literatura na área de metas e autogerenciamento, mas principalmente que estudos sobre a produção científica na área sejam desenvolvidos, pois ainda há questões a serem investigadas nesse sentido (VAN YPEREN, BLAGA \& POSTMES, 2014).

No Brasil as pesquisas em $\mathrm{CO}$ tem crescido de forma significativa nos últimos anos (BORGES-ANDRADE; PAGOTTO, 2010), mas apesar de sua relevância teórica, bem como de seus inúmeros desdobramentos práticos, não há uma agenda de pesquisa efetivamente estabelecida, o que torna a produção pontual e reduz o potencial de desenvolvimento da área. Assim, o presente estudo visa também colaborar no sentido de apontar a relevância da área de autogerenciamento e metas, que mesmo estando presente de forma significativa no cotidiano das organizações, tem muito pouco respaldo teórico na produção nacional.

Apesar da importância do campo, a produção brasileira em $\mathrm{CO}$, mesmo apresentando crescimento nos últimos anos (BORGES-ANDRADE; PAGOTTO, 2010), ainda enfrenta algumas dificuldades, especialmente metodológicas. Exemplos são a necessidade de melhoria de medidas, a padronização de alguns métodos, a otimização das análises de dados e a integração mais estreita com outras áreas do saber (GONDIM; BORGES-ANDRADE; BASTOS, 2017). Se considerarmos as revisões internacionais, as lacunas entre os estudos brasileiros e os estudos internacionais ficam ainda mais evidentes, já que várias tendências observadas fora do país não são vistas no Brasil, como o uso mais frequente de métodos inferenciais, análises multinível e a preocupação com a geração de instrumentos com uma qualidade psicométrica maior (JUDGE; KAMMEYER-MULLER, 2012).

As pesquisas em CO, e mais especificamente os construtos investigados, são estruturados em três grandes níveis: micro organizacional (indivíduos), meso-organizacional (equipes e grupos de trabalho) e macro organizacional (organização). Cada nível é caracterizado por uma predominância de métodos específicos e variáveis de interesse, ainda que sejam comuns os estudos que investiguem mais de um nível (BORGES-ANDRADE; PAGOTTO, 2010). Estudos recentes sobre a literatura internacional tem demonstrado um

Perspectivas em Gestão \& Conhecimento, João Pessoa, v. 8, n. 3, p. 243-254, set./dez. 2018. 
aumento significativo nos estudos em nível macro, geralmente com foco em organizações e equipes, ao mesmo tempo em que temas clássicos como liderança têm sido estudados em níveis múltiplos de análise (BORGES-ANDRADE; PEREZ; TORO, 2018).

No que se refere aos temas de pesquisa, enquanto internacionalmente há uma tendência de aumento dos estudos de temas ligados à cognição nas organizações (CÔTÉ, 2014; NOE; CLARKE; KLEIN, 2014; ZHOU; HOEVER, 2014) e mais precisamente, um aumento nas publicações investigando o autogerenciamento e as metas (BREEVAART; BAKKER; DEMEROUTI, 2014; BURNETTE; O'BOYLE, VANEPPS; POLLACK; FINKEL, 2013; KOCH; NAFZIGER, 2011; UNSWORTH; MASON, 2016), no Brasil essa área ainda é pouco explorada, como foi possível identificar nos resultados do presente artigo.

\section{MÉTODO}

Foi realizada uma revisão sistemática de literatura, a partir da elaborada por c e da atualização do banco de dados desta mesma pesquisa, que vem ocorrendo ao longo dos anos. Uma vez que esta fonte secundária de dados engloba o período entre 1996 e 2017, o presente estudo se fundamentou em artigos publicados em periódicos nacionais neste mesmo período de tempo. De acordo com revisões anteriores, um número ínfimo de publicações dentro do tema autogerenciamento e objetivos do trabalho encontradas antes de 1996 e seu aumento só se tornou significativo a partir dos anos 2000 , o que nos leva a conclusão de que a opção pelo escopo temporal parece adequada para o objetivo do presente artigo (FONSECA; PORTO; BORGES-ANDRADE, 2015).

O conjunto de periódicos da fonte de dados é composto pelas 19 revistas brasileiras de psicologia e administração, de reconhecida qualidade, que mais publicam estudos sobre comportamento organizacional. As revistas de psicologia são: Psicologia: Reflexão e Crítica (UFRS); Psicologia em Estudo (UEM); Estudos de Psicologia (PUC Campinas); Psicologia: Teoria e Pesquisa (UnB); Psico USF; Psico PUCRS; Psicologia, Ciência e Profissão (CFP); Psicologia: Organizações e Trabalho (SBPOT); Estudos de Psicologia (UFRN); Avaliação Psicológica (IBAP); Paidéia (USP Ribeirão Preto). As revistas de administração: Revista de Administração da Universidade de São Paulo (USP); Revista de Administração Pública (FGV/RJ); Organizações e Sociedade (UFBA); Revista de Administração Contemporânea (ANPAD); Revista de Administração de Empresas (FGV/SP); Brazilian Administration Review (ANPAD); Revista de Administração Mackenzie (U. Presb. Mackenzie); Revista Eletrônica de Administração (UFRGS).

Para a amostragem de artigos conforme o método que vem sendo utilizado desde a primeira análise realizada por Borges-Andrade e Pagotto (2010), foram escolhidos apenas relatos de pesquisas empíricas, no âmbito do comportamento organizacional (que tenham estudado ao menos uma variável neste escopo). A partir deste critério, estudantes de mestrado e de doutorado vêm avaliando os artigos das revistas em questão, os classificando de acordo com critérios predefinidos pelo próprio banco de dados. A escolha desse modelo de categorização segue o padrão já estabelecido em outras revisões de literatura semelhantes e apresenta vantagens em relação à busca por palavras-chave em bancos de dados (problemas de indexação que poderiam excluir alguns artigos), bem como garante a inclusão das principais revistas nas duas grandes áreas de pesquisa sobre o tema (psicologia e administração). Assim, permite uma análise das publicações mais relevantes sobre comportamento organizacional (BORGES-ANDRADE; PAGOTTO, 2010; FONSECA; PORTO; BORGES-ANDRADE, 2015)

Nessa classificação, autogerenciamento e objetivos no trabalho definem uma subcategoria dentro da categoria cognição no trabalho, que abarca ainda: percepção de justiça e equidade no trabalho; percepção de suporte; percepção de imagens ou metáforas organizacionais; e atribuição de causalidade. Por meio de uma caracterização dessa subcategoria, pretende-se comparar a produção nacional em autogerenciamento e objetivos

Perspectivas em Gestão \& Conhecimento, João Pessoa, v. 8, n. 3, p. 243-254, set./dez. 2018. 
no trabalho com os dados descritivos de pesquisas brasileiras no âmbito do $\mathrm{CO}$, pertencentes à mesma planilha, a qual não se limita ao tema de autogerenciamento e objetivos no trabalho, mas aborda todas as categorias de CO.

\section{RESULTADOS}

\subsection{Tradição nacional em autogerenciamento e objetivos no trabalho}

No levantamento realizado, considerando as publicações nacionais nas 19 principais revistas de administração e psicologia, no período entre 1996 e 2017, foram localizados 16 artigos sobre o tema autogerenciamento e objetivos no trabalho. Os artigos apresentaram uma subdivisão, entre análises no âmbito micro (comportamentos individuais) e no âmbito macro (carreira e mudança organizacional) (Tabela 1 ).

Tabela 1. Nível de análise da produção nacional sobre o tema autogerenciamento e objetivos no trabalho e seus subtemas.

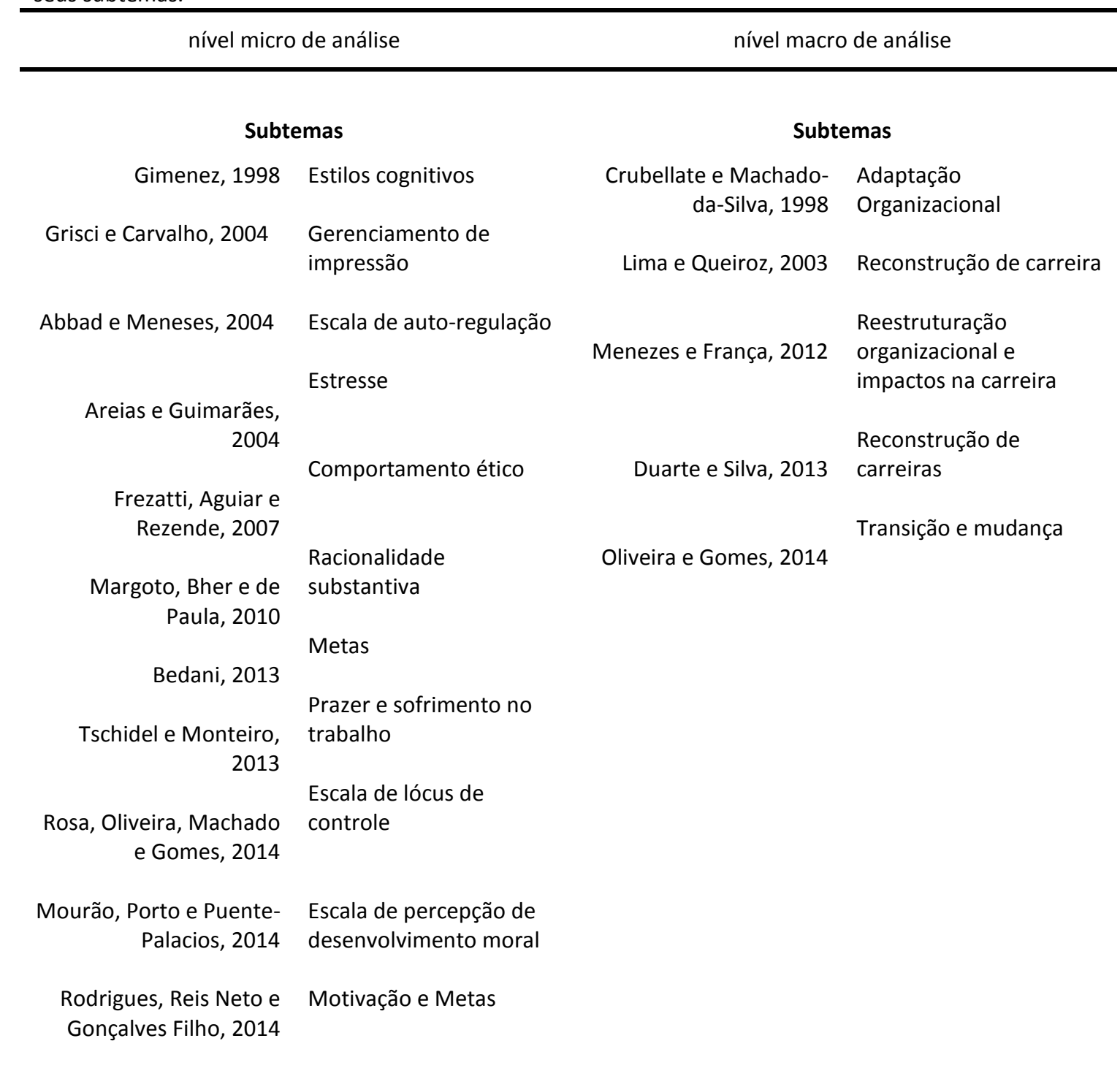

Fonte: Dados da pesquisa (2017)

Perspectivas em Gestão \& Conhecimento, João Pessoa, v. 8, n. 3, p. 243-254, set./dez. 2018. 
Nos artigos que abordaram o tema por uma análise em nível micro, os subtemas investigados variaram entre metas, gerenciamento de impressão, comportamento ético e estilos cognitivos (BEDANI, 2013; CARVALHO; GRISCI, 2003; FREZATTI; AGUIAR; REZENDE, 2007; GIMENEZ, 1998). Ainda neste âmbito, alguns trabalhos se direcionaram a validação de instrumentos (ABBAD; MENESES, 2004; MOURÃO; PORTO, PUENTE-PALACIOS, 2014; ROSA; OLIVEIRA; MACHADO; GOMES, 2014). Por sua vez, os artigos realizados em um nível macro de análise incluíram subtemas como: reconstrução de carreira; reestruturação organizacional e impactos na carreira; transição e mudanças; aposentadoria e demissão (LIMA; QUEIROZ, 2003; MENEZES; FRANÇA, 2012; OLIVEIRA; GOMES, 2014; DUARTE; SILVA, 2013). (Tabela 1)

A tabela 2 mostra os aspectos metodológicos dos estudos encontrados, evidenciando um caráter pouco pluralístico tanto em termos de desenho de pesquisa quanto em termos de análise de dados. Os artigos encontrados foram conduzidos por meio de desenhos de pesquisa em sua maioria do tipo com delineamento em detrimento de relatos de experiência. Dentre os delineamentos, foi encontrada a predominância de surveys, os quais majoritariamente utilizaram questionários e entrevistas. Estes instrumentos foram aplicados em amostragens primordialmente de empresas do setor público $(n=7,43,8 \%)$ e no segmento de economia terciário ( $n=11,68,8 \%)$.

No que se refere aos procedimentos de coleta e análise dos dados, foi observada a ausência de abordagens multimétodos, com uma preferência do uso exclusivo de análises quantitativas ou qualitativas. Em consonância com esse aspecto, os dados são quase que exclusivamente de origem primária, tratados por meio de análises inferenciais $(n=8,50 \%)$ e análise de conteúdo $(n=8,50 \%)$.

Tabela 2. Caracterização do método na produção nacional sobre autogerenciamento e objetivos no trabalho

\begin{tabular}{|c|c|c|}
\hline & Frequência & Porcentagem \\
\hline \multicolumn{3}{|l|}{ Natureza do estudo } \\
\hline Pesquisa com desenho & 14 & $87,50 \%$ \\
\hline Relato de experiência & 2 & $12,50 \%$ \\
\hline \multicolumn{3}{|l|}{ Desenho do estudo } \\
\hline Survey & 15 & $93,80 \%$ \\
\hline Experimental & 0 & $0 \%$ \\
\hline Pesquisa-ação & 1 & $6,30 \%$ \\
\hline \multicolumn{3}{|l|}{ Natureza da pesquisa } \\
\hline Quantitativa & 8 & $50 \%$ \\
\hline Qualitativa & 8 & $50 \%$ \\
\hline Qualitativa-quantitativa & 0 & $0 \%$ \\
\hline \multicolumn{3}{|l|}{ Finalidade da pesquisa } \\
\hline Gerar conhecimento & 13 & $81,30 \%$ \\
\hline Gerar instrumento & 2 & $12,50 \%$ \\
\hline Gerar conhecimento e instrumento & 1 & $6,30 \%$ \\
\hline Origem dos dados & & \\
\hline
\end{tabular}

Perspectivas em Gestão \& Conhecimento, João Pessoa, v. 8, n. 3, p. 243-254, set./dez. 2018. 
$\begin{array}{lll}\text { Primária } & 15 & 93,80 \%\end{array}$

Fonte: Dados da pesquisa (2017)

Por fim, cabe ressaltar que a maioria dos artigos apresentava como objetivo a geração de conhecimento sobre o tema em questão o qual, por sua vez, relaciona-se com construtos tipicamente mais associados ao âmbito da psicologia do que da administração. Em consonância, a maioria dos autores dos artigos foram psicólogos ( $n=27,67,7 \%)$ e doutores ( $n$ $=27,70,6 \%$ ).

\subsection{Tradição nacional em autogerenciamento e objetivos no trabalho vs. tradição nacional em comportamento organizacional}

No cenário brasileiro, a produção científica em CO está fortemente ligada aos grupos de pesquisa de pós-graduação. Linhas de pesquisa bem estruturadas não apenas facilitam a produção científica, mas proporcionam a formação de novos pesquisadores, que dão continuidade ao processo de crescimento e delimitação dos vieses de investigação em CO. No país, a pesquisa nesta área está, em sua maior parte, relacionada à psicologia social $(29,6 \%)$, seguida pelas psicologias clínica $(17,6 \%)$ e do desenvolvimento $(11,6 \%)$ (BORGES-ANDRADE; PAGOTTO, 2010). Esta distribuição poderia levar à conclusão de que a produção em autogerenciamento e objetivos no trabalho é robusta, já que a forte influência da psicologia social em CO poderia levar a uma investigação do estabelecimento de metas no cenário brasileiro, já que este tema é relevante em outras áreas, como a psicologia social. De fato, a maior parte dos autores que produziram conhecimento em autogerenciamento e objetivos no trabalho se caracterizaram como psicólogos doutores, portanto, possivelmente ligados às linhas de pesquisa em pós-graduação. No entanto, a produção sobre o tema (apenas 16 artigos) não refletiu a forte influência das linhas de pesquisa de pós-graduação, nem a preponderância da psicologia social nos estudos em CO.

Os temas de maior destaque nas publicações nacionais sobre CO são avaliação e medidas (20\%), seguidas do tema trabalho, identidade e subjetivação e do tema trabalho, saúde e gestão de pessoas, os quais, cada um, acrescentam $11 \%$ ao total da produção. Em especial, a predominância se dá em estudos com foco em afeto no trabalho, aprendizagem, interações sociais, bem-estar e saúde e identidade e significados (BORGES-ANDRADE; PAGOTTO, 2010). Assim, o panorama geral brasileiro já indica que o tema geral cognição no trabalho, bem como o subtópico autogerenciamento e objetivos no trabalho não se tratam de assuntos muito investigados na literatura.

Em confirmação a essa tendência, enquanto assuntos como afeto e aprendizagem apresentaram um total de publicações de 117 e 82 artigos, respectivamente, no período em investigação, a área de autogerenciamento e objetivos no trabalho apresentou, no mesmo período, somente 16 artigos. Apenas os temas fuga e esquiva (11 artigos), criatividade e solução de problemas (9 artigos) e motivação (12 artigos) demonstraram uma produção menor. Se considerarmos que a média de publicações em CO é de 40 artigos por ano (DP = 19,1), evidencia-se ainda mais a baixa produção na área em questão.

Em parte, este fenômeno poderia derivar do afastamento típico da área de $\mathrm{CO}$, entre a produção de conhecimento e a prática. O perfil de psicólogos doutores vinculados à pósgraduação, que produzem conhecimento sobre o tema autogerenciamento e objetivos no trabalho, não retrata o perfil dos profissionais na área de psicologia no Brasil. Segundo Neiva e Corradi (2011) os psicólogos brasileiros podem ser caracterizados como uma maioria de 
mulheres (83\%), residentes no sudeste (49\%) e atuando em escritórios, consultórios ou residências (37\%). Esses profissionais, por serem geralmente graduados (45\%) ou com especialização (39\%), denunciam a discrepância entre estudos aplicados e pesquisa básica em psicologia, realizada na academia.

No entanto, o tema da cognição no trabalho aparenta causar um grande interesse prático para aqueles que atuam com a gestão de pessoas. Juntamente com atitudes frente à mudança organizacional, aprendizagem, cultura organizacional e contratos psicológicos, a cognição no trabalho se trata de um dos principais temas de periódicos científicos de administração e não dos de psicologia (NEIVA; CORRADI, 2011).

Em termos de desenho de pesquisa, é possível que o padrão dos delineamentos aponte para uma área ainda em desenvolvimento, buscando construir conceitos antes de partir necessariamente para aplicações dos construtos em contextos específicos. A maior parte dos artigos publicados na área foi formada por estudos de pesquisa com delineamento $93 \%$ em detrimento de relatos de experiência, sendo que $81 \%$ tinham justamente como objetivo a geração de conhecimento. A forma de produzir este conhecimento, no entanto, aparenta estar aquém da área de $\mathrm{CO}$, na qual nota-se uma tendência para o uso de métodos e delineamentos de pesquisa mais complexos, bem como para estudos em níveis de análise macro. Um total de $48 \%$ das pesquisas em CO são quali-quanti. Já na área de autogerenciamento e objetivos no trabalho, apenas $12 \%$ se caracterizam pelo uso de ambos os métodos de análise. Artigos de perspectiva macro em autogerenciamento e objetivos no trabalho foram publicados apenas a partir de 2010, o que ao mesmo tempo em que contribui para a contemporaneidade dos dados, por outro lado indica a incipiência das pesquisas com essa característica.

Por outro lado, é possível observar convergências entre a área de $\mathrm{CO}$ e autogerenciamento e objetivos no trabalho, em algumas escolhas metodológicas. $O$ uso frequente de survey é difundido em ambas as áreas, com 92\% de pesquisas desse tipo em CO e $82,3 \%$ na área específica de autogerenciamento e objetivos no trabalho. Mais especificamente sobre as análises, em CO ainda vigora a predominância de métodos inferenciais (47\%), valor igual ao encontrado nas pesquisas sobre autogerenciamento e objetivos no trabalho. Nota-se ainda a mesma presença significativa de estudos no setor terciário em ambas as áreas (78\% dos estudos em CO, 64,7\% dos estudos em autogerenciamento e objetivos no trabalho).

Os dados indicam, portanto, uma produção ainda incipiente sobre o tema específico, mas já com algumas características do campo de comportamento organizacional se apresentando, fator que, caso haja um aumento nas publicações na área, pode se conformar em um padrão de semelhança ainda maior. $O$ aumento dessa produção, no entanto, perpassa questões de aproximação entre teoria e prática nos estudos, publicação e realidade da atuação em comportamento organizacional.

Outro aspecto importante e ainda pouco investigado, diz respeito a diferenças transculturais do fenômeno em questão. Nenhum dos artigos brasileiros abordou essa temática apesar da literatura internacional apontar que, por exemplo, culturas mais coletivistas tem um posicionamento de autogerenciamento e autorregulação diferente de culturais individualistas (DELLE FAVE; WISSING; BRDAR; VELLA-BRODRICK; FREIRE, 2009; KURMAN, 2001). Outros estudos cobre coletivismo e individualismo apontam ainda diferenças no âmbito de verticalidade e horizontalidade em termos de autogerenciamento e comportamento direcionado e metas (KRUGLANSKI et al., 2000; SHELDON et al., 2004).

\subsection{Agenda de pesquisa para autogerenciamento e objetivos no trabalho no Brasil}

A grande questão no que se refere aos estudos brasileiros sobre autogerenciamento e objetivos no trabalho é a incipiência. Apenas a partir de 2010 é possível afirmar que os trabalhos sobre o tema começaram a ser publicados de maneira mais significativa. Além disso,

Perspectivas em Gestão \& Conhecimento, João Pessoa, v. 8, n. 3, p. 243-254, set./dez. 2018. 
pela diversidade de autores, percebe-se que não se tratam de trabalhos que fazem parte necessariamente de uma agenda de pesquisa sobre o tema e sim, de temas tangenciais de agendas prévias dos pesquisadores. Neste ponto é que reside a principal mudança que deve ocorrer na área - a criação de uma agenda de pesquisa, que possa direcionar os pesquisadores no desenvolvimento de trabalhos que contribuam em uma mesma direção para o crescimento do tema no Brasil.

Se considerarmos o estado atual da pesquisa internacional, podemos identificar alguns pontos importantes para a agenda de pesquisa brasileira sobre autogerenciamento e objetivos no trabalho. Primeiramente, é necessário o desenvolvimento de instrumentos de pesquisa mais robustos e adequados aos aspectos socioculturais do país. Sem medidas confiáveis a tarefa de produzir conhecimento que possam contribuir para o crescimento da área é dificultada. Este objetivo caminha em convergência com o perfil da própria área de $\mathrm{CO}$, na qual desenvolvimento de medidas é um tema predominante.

Em segundo lugar, é possível sugerir o aprofundamento das pesquisas básicas por meio do estabelecimento de redes de pesquisa. Com uma produção prolífica como é a de comportamento organizacional e mesmo da psicologia organizacional como um todo, não é difícil o desenvolvimento de uma integração maior com grupos de pesquisadores que já produzam conhecimento os quais possam ser agregados ao tema específico de autogerenciamento e objetivos no trabalho.

Como terceiro ponto, Borges-Andrade et al. (2017) sugerem que a multiplicidade de formas de trabalho, tipos de empregados e formas de interação nos contextos organizacionais deve ser investigado em sua perspectiva transcultural, e o Brasil, com sua gama de grupos culturais, tem muito a contribuir nesse sentido. Finalmente, o desenvolvimento do campo requer ainda uma abertura dos periódicos para a publicação de artigos e trabalhos com a temática do autogerenciamento e metas no trabalho, o que historicamente já se sabe que contribuiu de forma significativa para o crescimento de quaisquer áreas de investigação.

\section{CONSIDERAÇÕES FINAIS}

Apesar de ser um assunto tão relevante para a compreensão dos fenômenos organizacionais, a pesquisa brasileira em autogerenciamento e objetivos no trabalho ainda necessita de um esforço mais intenso, não apenas para se adequar ao que já ocorre a nível internacional, mas especialmente para possibilitar um maior entendimento dos construtos que afetam o fenômeno a nível nacional.

No que se refere à psicologia organizacional, já temos uma estrutura de produção de conhecimento relativamente robusta, formada por programas de pós-graduação, núcleos de pesquisa e periódicos que possibilitam uma produção científica de excelente nível. Dessa forma, é tão somente uma questão de instigar, nos pesquisadores em formação e nos já formados, o interesse em desenvolver uma agenda de pesquisa conjunta sobre o tema.

O campo de estudo do autogerenciamento e objetivos no trabalho mostra, em nível internacional, uma capacidade de aplicabilidade e maior compreensão das questões que seria importante para o desenvolvimento científico no Brasil. Além disso, um maior interesse no tema por parte dos pesquisadores mais experientes atrairia pesquisadores mais jovens, que dariam prosseguimento aos estudos sobre autogerenciamento e objetivos no trabalho.

Em uma área com tamanho potencial inter e multidisciplinar, ainda vislumbra-se a exponenciação desses efeitos com a integração da agenda de pesquisa em psicologia organizacional com a pesquisa no campo da administração, bem como de outras áreas correlatas que, historicamente, contribuem para estudos em psicologia organizacional e comportamento organizacional.

Perspectivas em Gestão \& Conhecimento, João Pessoa, v. 8, n. 3, p. 243-254, set./dez. 2018. 


\section{REFERÊNCIAS}

ABBAD, G.; MENESES, P. P. M. Lócus de controle: validação de uma escala em situação de treinamento. Estudos de Psicologia, Natal, v. 9, n. 3, p. 441-450. 2004. http://doi.org/10.1590/S1413-294X2004000300006.

BEDANI, M. Os Desafios éticos na gestão de instituições financeiras de grande porte: A percepção de executivos do banco do Brasil. Psicologia: Organizações e Trabalho, v. 13, n. 1, p. 5-18. 2013.

BORGES-ANDRADE, J. E.; PAGOTTO, C. D. P. O estado da arte da pesquisa brasileira em Psicologia do Trabalho e Organizacional. Psicologia: Teoria e Pesquisa, v. 26, n. Especial, p. 3750. 2010. http://doi.org/10.1590/S0102-37722010000500004.

BORGES-ANDRADE, J. E.; PEREZ, E. R.; TORO, J. P. Organizational/Work Psychology in Latin America. In: ARDILA, R. (Ed.) Psychology in Latin America: Current status, challenges and perspectives. Switzerland: Springer, 2018, p. 105-158.

BREEVAART, K.; BAKKER, A. B.; DEMEROUTI, E. Daily self-management and employee work engagement. Journal of Vocational Behavior, v. 84, n. 1, 2014, p.31-38.

BURNETTE, J. L.; O'BOYLE, E. H.; VANEPPS, E. M.; POLLACK, J. M.; FINKEL, E. J. Mind-sets matter: A meta-analytic review of implicit theories and self-regulation. Psychological Bulletin, v. 139 , n. 3 , p. $655-701.2013$.

CARVALHO, M. L.; GRISCI, C. L. I. Gerenciamento de impressão e produção de subjetividade em entrevista de seleção. RAE Eletrônica, v. 2, n. 2, p. 72-85. 2013.

CAVALLO, J. V.; HOLMES, J. G.; FITZSIMONS, G. M.; MURRAY, S. L.; WOOD, J. V. Managing motivational conflict: How self-esteem and executive resources influence self-regulatory responses to risk. Journal of Personality and Social Psychology, v. 103, n. 3, p. 430-451. 2012.

CÔTÉ, S. (2014). Emotional intelligence in organizations. Annual Review of Organizational Psychology and Organizational Behavior, v. 1, n. 1, p. 459-488. 2014.

DE CAMPOS, M. I.; RUEDA, F. J. M. Regressão linear e quadrática: Análises comparativas de efeito em medidas do comportamento organizacional. Estudos de Psicologia, v. 22, n. 2, p. 225-234. 2017.

DELLE FAVE, A.; WISSING, M.; BRDAR, I.; VELLA-BRODRICK, D.; FREIRE, T. Cross-cultural perceptions of meaning and goals in adulthood: Their roots and relations with happiness. In: WATERMAN, A. S. (Ed.). The best within us: Positive psychology perspectives on eudaimonia. Washington DC: APA, 2009, p. 227-247.

FONSECA, A. M. D. O.; PORTO, J. B.; BORGES-ANDRADE, J. E. Liderança: Um retrato da produção científica brasileira. Revista de Administração Contemporânea, v. 19, n.1, p. 290310. 2015.

FREZATTI, F.; AGUIAR, A. B. DE; REZENDE, A. J. Respostas estratégicas às pressões institucionais e sucesso no atingir metas no orçamento: Um estudo em uma empresa multinacional.

Perspectivas em Gestão \& Conhecimento, João Pessoa, v. 8, n. 3, p. 243-254, set./dez. 2018. 
Organizações \& Sociedade, v. 14, n. 43, p. 141-158. 2007.

GELFAND, M. J. et al. Cross-cultural industrial organizational psychology and organizational behavior: A hundred-year journey. Journal of Applied Psychology, v. 102, n. 3, p. 514. 2017. GIMENEZ, F. A. P. Escolhas estratégicas e estilo cognitivo: um estudo com pequenas empresas. Revista de Administração Contemporânea, v. 2, n. 1, p. 27-45. 1998.

GONDIM, S. M. G.; BORGES-ANDRADE, J. E.; BASTOS, A. V. B. Psicologia do trabalho e das organizações: Produção científica e desafios metodológicos. Revista Psicologia em Pesquisa, $v$. 4, n. 2. p.84-99. 2017.

HANNECK, C. J.; SANTOS, P. K. A importância do comportamento organizacional de maneira eficaz em nível individual e em grupo. Brazilian Journal of Development, v. 4, n. 3, p. 671-686. 2018.

JENSEN, J. M.; RAVER, J. L. When self-management and surveillance collide: Consequences for employees' organizational citizenship and counterproductive work behaviors. Group \& Organization Management, v. 37, n. 3, p. 308-346. 2012.

JUDGE, T. A.; KAMMEYER-MUELLER, J. D. Job Attitudes. Annual Review of Psychology, v. 63, n. 1, p. 341-367. 2012.

$\mathrm{KOCH}$, A. K.; NAFZIGER, J. Self-regulation through goal setting. Scandinavian Journal of Economics, v. 113, n. 1, p. 212-227. 2011.

KRUGLANSKI, A W.; THOMPSON, E. P.; HIGGINS, E. T.; ATASH, M. N.; PIERRO, A; SHAH, J. Y.; SPIEGEL, S. To "do the right thing" or to "just do it": locomotion and assessment as distinct self-regulatory imperatives. Journal of Personality and Social Psychology, v. 79, n. 5, p. 793815. 2000.

KURMAN, J. Self-regulation strategies in achievement settings: Culture and gender differences. Journal of Cross-Cultural Psychology, v. 32, n. 4, p. 491-503. 2001.

LIMA, M. C. R.; QUEIROZ, S. S. Organizações que mudam, vidas que se transformam: a posição dos sujeitos diante de processos de reestruturação organizacional na administração pública. Revista Psicologia: Organizações e Trabalho, v. 3, n. 1, p. 89-110. 2003.

LORD, R. G.; DIEFENDORFF, J. M.; SCHMIDT, A. M.; HALL, R. J. Self-regulation at work. Annual Review of Psychology, v. 61, p. 543-568. 2010.

MENEZES, G. S.; FRANÇA, L. H. Preditores da decisão da aposentadoria por servidores públicos federais. Revista Psicologia Organizações e Trabalho, v. 12, n. 3, p. 315-328. 2012.

MOURÃO, L.; PORTO, J. B.; PUENTE-PALACIOS, K. Construção e evidências de validade de duas escalas de percepção de desenvolvimento profissional. Psico-USF, v. 19, n. 1, p. 73-85. 2014.

NEIVA, E. R.; CORRADI, A. A. A psicologia organizacional e do trabalho no brasil: Uma análise a partir das redes sociais de pesquisadores da pós-graduação. Psicologia: Organizações e Trabalho, v. 10, n. 2, p. 67-84. 2011. 
NOE, R. A.; CLARKE, A. D. M.; KLEIN, H. J. Learning in the twenty-first-century workplace. Annual Review of Organizational Psychology and Organizational Behavior, v. 1, n. 1, p. 245275. 2014.

OLIVEIRA, M. Z. DE; GOMES, W. B. Estilos reflexivos e atitudes de carreira proteana e sem fronteiras nas organizações contemporâneas brasileiras. Revista Psicologia Organizações e Trabalho, v. 14, n. 1, p. 105-118. 2014.

PODSAKOFF, P. M.; MACKENZIE, S. B.; PODSAKOFF, N. P. (Ed.). The Oxford Handbook of Organizational Citizenship Behavior. Oxford University Press, 2018

ROSA, A. M. T. DA; OLIVEIRA, M. Z. DE; MACHADO, W. D. L.; GOMES, W. B. Development of the self-regulatory scale in work-related interactions. Psicologia: Reflexão e Crítica, v. 27, n. 2, p. 308-316. 2014.

SHELDON, K. M.; ELLIOT, A. J.; RYAN, R. M.; CHIRKOV, V.; KIM, Y.; WU, C., ... SUN, Z. SelfConcordance and subjective well-being in four cultures. Journal of Cross-Cultural Psychology, v. 35, n. 2, p. 209-223. 2004.

SOBRAL, F. J. B. D. A.; MANSUR, J. A. Produção científica brasileira em comportamento organizacional no período 2000-2010. Revista de Administração de Empresas, v. 53, n. 1, p. 21-34. 2013.

STURGES, J.; CONWAY, N.; LIEFOOGHE, A. Organizational support, individual attributes, and the practice of career self-management behavior. Group \& Organization Management, v. 35, n. 1, p. 108-141. 2010.

UNSWORTH, K. L.; MASON, C. M. Self-concordance strategies as a necessary condition for self-management. Journal of Occupational and Organizational Psychology, v. 89, n. 4, p. 711733. 2016.

VAN YPEREN, Nico W.; BLAGA, Monica; POSTMES, Tom. A meta-analysis of self-reported achievement goals and nonself-report performance across three achievement domains (work, sports, and education). PloS one, v. 9, n. 4, p. e93594, 2014.

VELOSO, E. F. R.; DUTRA, J. S. Carreiras sem fronteiras na gestão pessoal da transição profissional: um estudo com ex-funcionários de uma instituição privatizada. Revista de Administração Contemporânea, v. 15, n. 5, p. 834-854. 2011.

ZHOU, J.; HOEVER, I. J. Research on workplace creativity: a review and redirection. Annual Review of Organizational Psychology and Organizational Behavior, v. 1, n. 1, p. 333-359. 2014.

Artigo recebido em 02/04/2017 e aceito para publicação em 27/11/2018 